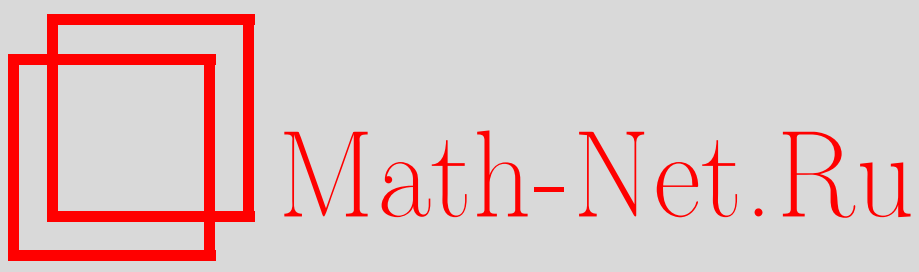

С. В. Пчелинцев, О тождествах свободных конечно порожденных альтернативных алгебр над полем характеристики 3, Матем. сб., 2001, том 192, номер 9, 109-124

DOI: https://doi.org/10.4213/sm596

Использование Общероссийского математического портала Math-Net.Ru подразумевает, что вы прочитали и согласны с пользовательским соглашением

http://www . mathnet.ru/rus/agreement

Параметры загрузки:

IP: 54.147 .182 .235

26 апреля 2023 г., 16:11:06 
УДК 512.554 .5

\section{С.В. Пчелинцев}

\section{О тождествах свободных конечно порожденных альтернативных алгебр над полем характеристики 3}

В 1981 году В.Т. Филиппов получил положительное решение проблемы Шестакова о строгости включений в цепочках многообразий, порожденных свободными альтернативньми и мальцевскими алгебрами конечного ранга над полем характеристики, отличной от 2 и 3 . В работе доказан аналогичньй результат для альтернативных алгебр над полем характеристики 3. Доказательство основано на построении трех серий тождеств, вьполняющихся в алгебрах соответствующего ранга. Опровержение тождеств на алгебрах большего ранга проводится с помощью первичной коммутативной альтернативной алгебры.

Кроме того, доказано, что в многообразиях альтернативных алгебр над полем характеристики 3 конечного базисного ранга всякая разрешимая алгебра нильпотентна.

Библиограффия: 9 названий.

\section{Введение}

В 1977 году И.П. Шестаков [1] доказал, что тождества свободных альтернативных алгебр над полем $\Phi$ характеристики 0 зависят от их ранга (ранг свободной алгебры - это минимальное число ее порождающих). Им же был поставлен вопрос о строгости включений в цепочке многообразий

$$
\mathrm{Alt}_{1} \Phi \subset \mathrm{Alt}_{2} \Phi \subset \cdots \subset \mathrm{Alt}_{n} \Phi \subset \cdots,
$$

порожденных свободными альтернативными $\Phi$-алгебрами конечного ранга. Положительное решение проблемы Шестакова в 1981 году получил В. Т. Филиппов [2] при условии, что кольцо операторов $\Phi$ содержит $\frac{1}{6}$.

Целью настоящей работы является доказательство аналогичного результата для альтернативных алгебр над полем характеристики $3^{1}$. Работа состоит из пяти параграфов. В $\S 1$ вводится понятие почти муфанговой функции и дается способ построения таких функций; затем определяются почти муфанговы функции типа $G_{n}$ и $F_{n}$. В $\S 2$ формулируется основная лемма, позволяюшая с помошью почти муфанговых функций указывать тождества алгебр заданного ранга $-G_{n}, F_{n}$ и $H_{n}$ (для подходящих значений $n$ ). В $\S 3$ устанавливается, что в исключительной первичной коммутативной альтернативной алгебре нет тождеств типа $G_{n}, F_{n}$ и $H_{n}$.

Работа вьполнена при поддержке Российского фонда фундаментальных исследований (грант № 0001-00339).

${ }^{1}$ Небольшой модификацией рассуждений последнее требование можно было бы заменить условием "кольцо $\Phi$ содержит $\frac{1}{2}$ ”, однако мы этого делать не будем, чтобы не усложнять доказательств.

(C) С. В. Пчелинцев 2001 
На основании этих результатов в $\S 4$ доказывается теорема о строгости включений в цепочке многообразий (*) алгебр над полем $\Phi$ характеристики 3 . Наконец, в $\S 5$

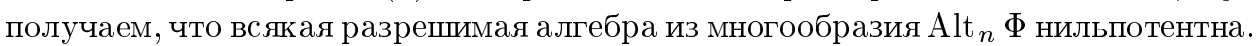

\section{§1. Вспомогательные функции}

1.1. Обозначения. Предварительные замечания. На протяжении всей работы используются следующие обозначения: $A$ - альтернативная алгебра над полем характеристики 3, т.е. алгебра, в которой ассоциатор $(x, y, z):=$ $(x y) z-x(y z)$ является кососимметрической функцией своих аргументов; $A^{(+)}$ и $A^{(-)}$- присоединенные йорданова и мальцевская алгебры, умножения в которых получены соответственно симметризацией и кососимметризацией умножения в алгебре $A: x \circ y:=x y+y x ;[x, y]:=x y-y x$; операторы $R_{x}, R_{x}^{(+)}, R_{x}^{(-)}$обозначают операторы правого умножения на элемент $x$ в алгебрах $A, A^{(+)}, A^{(-)}$соответственно. Напомним, что операторы $R_{x}^{(-)}, R(x, y):=R_{x} R_{y}-R_{x y}, D(x, y):=$ $\left[R_{x}^{(+)}, R_{y}^{(+)}\right]$являются дифференцированиями присоединенной йордановой алгебры $A^{(+)}$.

Все недостающие определения и обозначения можно найти в книге [3].

Следуя работе [4], введем операторные функции:

$$
\begin{aligned}
\left\{x_{1}, x_{2}, x_{3}\right\}_{c} & =(\mathbf{I d}+(13)+(23)) D\left(x_{1}, x_{2}\right) R\left(x_{3}, c\right), \\
\nabla_{a}\left(x_{1}, x_{2}, x_{3}\right) & =(\mathbf{I d}+(13)+(123)) R\left(a, x_{1}\right) D\left(x_{2}, x_{3}\right),
\end{aligned}
$$

где в скобках перед операторами записаны подстановки, действующие, как обычно, на индексы (Id обозначает единичную подстановку), например:

$$
(\mathbf{I d}+(123)) D\left(x_{1}, x_{2}\right) R\left(x_{3}, c\right)=D\left(x_{1}, x_{2}\right) R\left(x_{3}, c\right)+D\left(x_{2}, x_{3}\right) R\left(x_{1}, c\right) .
$$

Далее, напомним, что функция $f(x, y)$ называется муфанговой относительно $x$ и $y$, если она по этим переменным линейна, кососимметрична и удовлетворяет тождеству

$$
f(x T(y), y)=f(x, y) T^{*}(y), \quad \text { где } T \in\{R, L\} \quad \text { и } \quad R^{*}=L, \quad L^{*}=R .
$$

В общем случае функция $f\left(x_{1}, \ldots, x_{n}\right)$ назьвается муфанговой относительно набора переменных $x_{1}, \ldots, x_{n}$, если она является муфанговой относительно любых двух переменных из этого списка. Центральный пример муфанговой функции в альтернативных алгебрах - ассоциатор.

Нам потребуется несколько вспомогательных утверждений. Первые две леммы доказаны в работе [4].

ЛЕмма 1. Во всякой альтернативной алгебре следующие функиии являются муфанговыми относительно переменных $x, y, z, t, v$ :

(a) $\{x, y, a\}_{z}$;

(б) $R(x, a)\{y, z, a\}_{t}$;

(в) $g(5):=g_{a}(x, y, z, t):=[x, a]\{y, z, a\}_{t}$;

(г) $G(6):=G_{a}(x, y, z, t, v):=(x, y, a)\{z, t, a\}_{v}$. 
ЛЕмма 2. Во всякой альтернативной алгебре верно соотношение:

$$
R(x, a)\{y, z, a\}_{t}=\nabla_{x}(y, z, a) R(a, t) .
$$

ЛЕМма 3. Пусть в произвольной альтернативной алгебре функция $f(v)$ линейна по переменной $v$ и удовлетворяет тождеству

$$
f(v) D(v, q) R(v, s)=0 .
$$

Tогда

$$
f(v)\{p, q, v\}_{s}=f(v) D(p, v) R(q, s)-f(p) D(v, q) R(v, s)
$$

ДокАЗАтЕЛьство. Линеаризация тождества (1) имеет вид:

$$
f(p) D(v, q) R(v, s)+f(v) D(p, q) R(v, s)+f(v) D(v, q) R(p, s)=0,
$$

т.е.

$$
f(v) D(p, q) R(v, s)=-f(p) D(v, q) R(v, s)-f(v) D(v, q) R(p, s) .
$$

Используя это тождество, преобразуем выражение:

$$
\begin{aligned}
f(v)\{p, q, v\}_{s}= & f(v)(D(p, q) R(v, s)+D(v, q) R(p, s)+D(p, v) R(q, s)) \\
= & -f(p) D(v, q) R(v, s)-f(v) D(v, q) R(p, s) \\
& +f(v) D(v, q) R(p, s)+f(v) D(p, v) R(q, s) \\
= & f(v) D(p, v) R(q, s)-f(p) D(v, q) R(v, s) .
\end{aligned}
$$

1.2. Почти муфанговы функции. В дальнейшем важную роль играют почти муфанговы функции, которые вводятся следующим образом.

ОПРЕДЕЛЕНИЕ. Пусть $f\left(x_{1}, \ldots, x_{k-1} \mid x_{k}, \ldots, x_{n}\right)$ - однородная функция, зависяшая только от указанных переменных, причем по переменным $x_{1}, \ldots, x_{k-1}$ она имеет степень 2 , а по остальным переменным линейна. Переменные $x_{1}, \ldots, x_{k-1}$

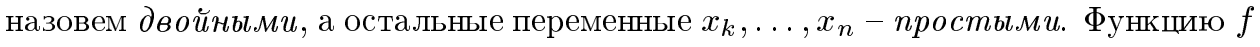
назовем почти муфанговой, если вьполнены следующие три условия:

(a) число ее простых переменных $\geqslant 4$ и по ним $f$ кососимметрична;

(б) функция $f$ обрашается в нуль, если вместо простой переменной подставить двойную переменную;

(в) по простым переменным функция $f$ муфангова.

$\mathrm{K}$ настоящему времени известны две почти муфанговы функции - это функция Филиппова $g(a \mid x, y, z, t)$ и ее аналог $G(a \mid x, y, z, t, v)$, построенные в рабо$\operatorname{Tax}[2],[4]$.

ЛЕмма 4 (достаточное условие почтимуфанговости). Если функиия $f$ удовлетворяет условиям (а) $и$ (б) определения и выполнены тожсдества $f\left(x_{1}, \ldots\right.$, $\left.x_{k-1} \mid x_{k}, \ldots, x_{n} T\left(x_{n-1}\right)\right)=f\left(x_{1}, \ldots, x_{k-1} \mid x_{k}, \ldots, x_{n}\right) T^{*}\left(x_{n-1}\right)$, то она является почти муфанговой и удовлетворяет следующим $M$-тожсдествам:

$$
f\left(x_{1}, \ldots, x_{k-1} \mid x_{k}, \ldots, x_{n} T\left(x_{i}\right)\right)=f\left(x_{1}, \ldots, x_{k-1} \mid x_{k}, \ldots, x_{n}\right) T^{*}\left(x_{i}\right), \quad i<n .
$$


ДокАЗАТЕЛЬСтво. Линеаризация исходного тождества

$$
f\left(x_{1}, \ldots, x_{k-1} \mid x_{k}, \ldots, x_{n} T\left(x_{n-1}\right)\right)=f\left(x_{1}, \ldots, x_{k-1} \mid x_{k}, \ldots, x_{n}\right) T^{*}\left(x_{n-1}\right)
$$

подстановкой $x_{n-1} \rightarrow x_{i}(i<n)$ приводит к тождеству

$$
\begin{aligned}
& f\left(x_{1}, \ldots, x_{k-1} \mid x_{k}, \ldots, x_{n-2}, x_{i}, x_{n} T\left(x_{n-1}\right)\right)+f\left(x_{1}, \ldots, x_{k-1} \mid x_{k}, \ldots, x_{n} T\left(x_{i}\right)\right) \\
& =f\left(x_{1}, \ldots, x_{k-1} \mid x_{k}, \ldots, x_{n-2}, x_{i}, x_{n}\right) T^{*}\left(x_{n-1}\right) \\
& \quad+f\left(x_{1}, \ldots, x_{k-1} \mid x_{k}, \ldots, x_{n}\right) T^{*}\left(x_{i}\right) .
\end{aligned}
$$

Замечая, что первые слагаемые в обеих частях этого равенства нулевые (по определению), получаем требуемое $M$-тождество.

ОПРЕДЕЛЕНИЕ. Линейную функцию $f(x)$ назовем $D$-функиией, если она является дифференцированием присоединенной йордановой алгебры $A^{(+)}$.

Заметим, что каждая муфангова функция $f(x, y)$ является $D$-функцией. В самом деле, во-первых, $f\left(x^{2}, x\right)=f(x, x) x=0$, откуда линеаризацией по переменной $x$ получаем $f(x \circ y, x)+f\left(x^{2}, y\right)=0$; воспользовавшись теперь свойством муфанговости, имеем $f\left(x^{2}, y\right)=-f(x \circ y, x)=-f(y, x) \circ x=f(x, y) \circ x$.

ЛЕмма 5. Если функиия $f(x, y)$ является $D$-функиией по каждой переменной, то она удовлетворяет тождеству $f(x, y) D_{x, y}=0$.

ДоказАТЕЛЬство. Имеем:

$$
\begin{aligned}
& f(x, y) R_{x}^{(+)} R_{y}^{(+)}-f(x, y) R_{y}^{(+)} R_{x}^{(+)}=f\left(x^{2}, y\right) R_{y}^{(+)}-f\left(x, y^{2}\right) R_{x}^{(+)} \\
& \quad=f\left(x^{2}, y^{2}\right)-f\left(x^{2}, y^{2}\right)=0 .
\end{aligned}
$$

Укажем простой способ построения почти муфанговых функций, сделав предварительно одно замечание.

Лемма 6. Пусть $f\left(B_{n} \mid P_{m}\right)$ - почти муфангова функиия, зависящая от двойных переменных из множества $B_{n}$ и линейных переменных из множества $P_{m} \quad(m \geqslant 4)$, и допустим, что функиия $f(u, v):=f\left(B_{n} \mid P_{m-2}, u, v\right)$ находится в условиях леммы 3, т.е. удовлетворяет тохсдетву (1). Тогда функия

$$
f\left(B_{n+1} \mid P_{m+2}\right)=f\left(B_{n} \mid P_{m-2}, p, b\right) \cdot\{q, s, b\}_{t},
$$

$2 \partial e$

$$
B_{n+1}=B_{n} \cup\{b\}, \quad P_{m+2}=P_{m-2} \cup\{p, q, s, t\},
$$

также является почти муфанговой. 
ДокАЗАТЕЛЬСтво. В силу лемм 3 и 5 имеем

$$
\begin{aligned}
f(p, v)\{p, q, v\}_{s} & =f(p, v) D(p, v) R(q, s)-f(p, p) D(v, q) R(v, s) \\
& =f(p, v) D(p, v) R(q, s)=0
\end{aligned}
$$

поскольку по условию функция $f(p, v)$ муфангова относительно $p, v$, а значит, $D$-функция. Следовательно, $f(p, v)\{q, s, v\}_{t}$ кососимметрична по $p, q, s, t$. Отсюда ввиду леммы 1 вытекает, что функция $f\left(B_{n+1} \mid P_{m+2}\right)$ является муфанговой относительно переменных из множества $P_{m+2}$. Наконец, если вместо простой переменной подставить двойную переменную, то получим нуль: действительно, ввиду кососимметричности $f(p, v)\{q, s, v\}_{t}$ по простым переменным достаточно для функции $f(p, v)\{q, s, v\}_{t}$ рассмотреть подстановку $p=v$ и получить очевидным образом равенство $f(v, v)=0$.

\section{СлЕДСТВИЕ. Функиия}

$$
g(8):=g(a, b \mid x, y, z, p, q, s):=g_{a}(x, y, z, b)\{p, q, b\}_{s}
$$

является почти муфанговой.

Лемма 7. Если $f(x, y, z, t)$ - муфангова функиия относительно переменных $x, y, z, t$, то справедливъ следующие свойства:

(a) функиия $h_{a}(x, y, z, t):=f(x, y, z, a) R(a, t)$ является D-функцией относительно переменных $x, y, z, t$ и кососимметрична по әтим переменHblм;

(б) $G_{a}(f(x, y, z, t), x, y, u, v)=0$.

ДокАЗАТЕльство. (а) Проверим сначала кососимметричность функции $h_{a}$ по указанньп переменным. В силу муфанговости функции $f$ имеем:

$$
\begin{aligned}
f(x, y, z, a) R(a, y) & =(f(x, y, z, a), a, y)=-(a, f(x, y, z, a), y) \\
& =-f(x, y, z, a) L(a) R(y)+f(x, y, z, a) R(y) L(a) \\
& =-f(x a, y, z, a) R(y)+f(x, y, y z, a) L(a) \\
& =-f(x a, y, y z, a)+f(x a, y, y z, a)=0 .
\end{aligned}
$$

Поскольку ассоциатор является $D$-функцией, то $D$-функцией является и функция $h_{a}(x, y, z, t)$ относительно указанных переменных.

(б) Имеем:

$$
\begin{aligned}
G_{a}( & f(x, y, z, t), x, y, u, v)=f(x, y, z, t) R(x, a)\{y, u, a\}_{v} \\
= & f(x, y, z, t) \nabla_{x}(y, u, a) R(a, v) \quad(\text { на основании леммы } 2(б)) \\
= & f(x, y, z, t)(R(x, y) D(u, a)+R(x, a) D(u, y)+R(x, u) D(a, y)) R(a, v) \\
= & f(x, y, z, t)(R(x, a) D(u, y)+R(x, u) D(a, y)) R(a, v) \quad \text { (в силу п. }(\mathrm{a})) \\
= & f(x, y, z, t)(-R(x, a) D(a, y) R(u, v)) \quad(\text { ввиду линеаризации по } a \\
& \quad \text { соотношения } R(x, a) D(a, y) R(a, v)=0[4 ; \text { лемма 2]) } \\
= & f(x, y, z, t) R(x, a) D(y, a) R(u, v)=0
\end{aligned}
$$

(в силу п. (a) функция $f(x, y, z, t) R(x, a)$ является $D$-функцией по переменньп $y, a$, значит, $(f(x, y, z, t) R(x, a)) D(y, a)=0)$. 
1.3. Вспомогательные функции типа $G$ и $F$. Пусть заданы множества

$$
B_{n}=\left\{b_{1}, b_{2}, \ldots, b_{n}\right\}, \quad P_{2 n}=\left\{p_{1}, \ldots, p_{2 n}\right\}
$$

Введем индукцией по $n(n \geqslant 1)$ функции $G(3 n+3):=G\left(B_{n} \mid P_{2 n+3}\right)$ :

$$
\begin{aligned}
G(6) & :=G_{b_{1}}\left(p_{1}, p_{2}, x, y, z\right), \\
G(3 n+6) & :=G\left(B_{n} \mid P_{2 n}, p_{2 n+1}, p_{2 n+2}, b_{n+1}\right) \cdot\left\{x, y, b_{n+1}\right\}_{z} \quad(n \geqslant 1) .
\end{aligned}
$$

Заметим, что функция $G(3 n+3)=G\left(B_{n} \mid P_{2 n}, x, y, z\right)$ имеет степень 2 по каждой переменной из множества $B_{n}$, а относительно каждой простой переменной эта функция линейна.

Наряду с функциями типа $G(n)$ введем еше одну серию функций:

$$
\begin{aligned}
F(3 n+7) & =F\left(B_{n}, a, c \mid P_{2 n}, x, y, z, t, v\right) \\
& =G_{a}\left(G\left(B_{n} \mid P_{2 n}, x, y, c\right), c, z, t, v\right) \quad(n \geqslant 1) .
\end{aligned}
$$

Лемма 8. Функиии $G(3 n+3), F(3 n+7)$ являются почти муфанговыми.

ДокАЗАТЕЛЬСтво. Функция $G(3 n+3)$ почти муфангова в силу леммы 6 . Для проверки почти муфанговости функции $F(3 n+7)$ достаточно заметить, что она обрашается в нуль при $y=z$, что немедленно вытекает из леммы 7 (б).

\section{§2. Основная лемма}

Почти муфанговы функции являются тождествами конечно порожденных алгебр. Более точно, справедлива

Лемма 9. Пусть $f_{n}$ - почти муфангова функция от $n$ переменных. Тогда справедливы следующие утверждения:

(а) функция $f_{n}$ является тождеством альтернативной алгебрь ранza $n-1$

(б) если число простых переменных почти муфанговой функиии $f_{n}$ не меньше 5, то функиия $\left(f_{n}, u, v\right)$ является тождеством альтернативной алгебры ранга $n$.

ДокаЗАтельство. (а) Докажем, что функция $f_{n}\left(x_{1}, \ldots, x_{k-1} \mid x_{k}, \ldots, x_{n}\right)$ обрашается в нуль в альтернативной $\Phi$-алгебре ранга $m:=n-1$. Для этого достаточно проверить, что верно тождество

$$
f_{n}\left(x_{1}, \ldots, x_{k-1} \mid x_{k}, \ldots, x_{m}, w\left(x_{1}, \ldots, x_{m}\right)\right)=0
$$

где $w\left(x_{1}, \ldots, x_{m}\right)$ - произвольный многочлен от указанных переменных.

Проведем индукцию по степени одночлена $w:=w\left(x_{1}, \ldots, x_{m}\right)$. Основание индукции при $\operatorname{deg} w=1$ следует из пп. (б) и (в) определения почти муфанговой функции. Для доказательства индукционного перехода заметим, что одночлен $w$ является линейной комбинацией одночленов той же степени, что и $w$, вида $a T(b) \cdots T(c)$, где $a, b, \ldots, c$ - элементы из множества $\left\{x_{1}, \ldots, x_{m}\right\}$ порождающих алгебры. К каждому из одночленов вида $a T(b) \cdots T(c)$ применимо подходящее 
$M$-тождество, значит, на основании индуктивного предположения получаем требуемое равенство (2) и для одночлена $w$.

(б) Пусть $A=\operatorname{Alt}\left[x_{1}, \ldots, x_{n}\right]$ - свободная альтернативная алгебра с множеством $\left\{x_{1}, \ldots, x_{n}\right\}$ свободных порождающих.

Докажем индукцией по степени $\operatorname{deg}\left(w_{1} w_{2}\right)$, что для любых одночленов $w_{1}, w_{2}$ от переменных $x_{1}, \ldots, x_{n}$ верно тождество

$$
\left(f_{n}, w_{1}, w_{2}\right)=0
$$

Основание индукции верно в силу леммы 7 (а). Сделав индуктивное предположение и воспользовавшись правым линеаризованным тождеством Муфанг

$$
\left(f_{n}, a, b c\right)+\left(f_{n}, b, a c\right)=\left(f_{n}, a, c\right) b+\left(f_{n}, b, c\right) a
$$

получаем, что без ограничения общности можно считать $\operatorname{deg} w_{1}=1$.

Обозначим через $I_{2}(A)$ линейное подпространство над полем $\Phi$, порожденное в алгебре $A$ квадратами всех элементов. Известно (см., например, [3; гл. 6, 1$]$ ), что $I_{2}(A)$ - идеал в алгебре $A$ и факторалгебра $A / I_{2}(A)$ нильпотентна индекса 4 . Отсюда в силу индуктивного предположения и тождества $\left(f_{n}, a, b^{2}\right)=\left(f_{n}, a, b\right) \circ b$ получаем, что одночлен $w_{2}$ можно считать полилинейным длины $\leqslant 3$. Кроме того, без ограничения общности мы можем считать, что переменная $w_{1}$ не входит в состав одночлена $w_{2}$.

Теперь для завершения доказательства тождества (3) осталось проверить равенство $\left(f_{n}, x_{n}, w\right)=0$, где $w$ - одночлен степени $\leqslant 3$, не содержащий переменной $x_{n}$. Снова в силу леммы 7 (а) функция $f(\cdot \mid \cdot, x, y, z, t, u) R(u, v)$ кососимметрична по переменным $x, y, z, t, v$, следовательно, без ограничения общности можно считать, что одночлен $w$ имеет вид $w=(x y) z$ (случай, когда в одночлен $w$ входит двойная переменная, легко получается линеаризацией). Тогда $f(\cdot \mid \cdot, x, y, z, t, u) R(u, w)=-f(\cdot \mid \cdot, x, y, z, w, u) R(u, t)$ и достаточно проверить, что $f(x, y, z, w):=f(\cdot \mid \cdot, x, y, z, w, u)=0$. Имеем:

$$
f(x, y, z, w)=f(x, y, z,(x y) z)=f(x, y, z, x) L(y) L(z)=0 .
$$

Итак, мы доказали, что

$$
\left(f_{n}, x_{n}, w\right)=0, \quad \text { где } \operatorname{deg} w \leqslant 3 \text { и } x_{n} \text { не входит в состав } w .
$$

Линеаризации последних тождеств подстановками $x_{n} \rightarrow x_{i}(i \neq n)$ показывают, что справедливы тождества $\left(f_{n}, x_{i}, w\right)=0$, где $i=1, \ldots, n ; \operatorname{deg} w \leqslant 3$. Тем самым тождества (3) доказаны.

\section{§ 3. О значениях многочленов $G_{n}, F_{n}, H_{n}$ на первичных коммутативных алгебрах}

Всюду в этом параграфе $S$ - первичная исключительная коммутативная альтернативная ниль-алгебра индекса 3 над полем характеристики 3 ; сушествование таких алгебр доказано в [5]. Пусть

$H(3 n+8):=H\left(B_{n}, a, c, d \mid P_{2 n}, x, y, z, t, v\right):=F\left(B_{n}, a, c \mid P_{2 n}, x, y, z, t, d\right) R(d, v)$. 
Докажем, что в алгебре $S$ не может вьполняться ни одно из тождеств вида

$$
H(3 n+8)=0 .
$$

Попутно, очевидно, будет доказано, что многочлены $G, F$ также ненулевые.

\section{1. Слабо муфанговы функции.}

ОПРЕДЕЛЕНИЕ. Функция $f(x, y)$, линейная по переменным $x, y$, называется $c л a$ бо муфанговой (на алгебре $S$ ), если на $S$ выполнены тождества:

$$
f(x, x)=0, \quad f\left(x^{2}, x\right)=0 .
$$

ЗАмечАниЕ. Всякая слабо муфангова функция $f(x, y)$ на алгебре $S$ удовлетворяет тождествам:

(a) $f(x, y)=-f(y, x)$ - кососимметричность;

(б) $f\left(x^{2}, y\right)=f(x y, x)$;

(в) $f(x y, z)=f(x, y z)+f(y, x z)$.

Действительно, указанные тождества получаются линеаризацией определяющих тождеств с учетом коммутативности алгебры $S$ и условия на ее характеристику: $\operatorname{char} S=3$.

Лемма 10. Пусть $f(x, y)$ - слабо муфангова функиия на алгебре $S$. Тогда

(a) $f\left(\left(x_{1}, x_{2}, x_{3}\right), x_{4}\right)=((14)+(24)+(34)) f\left(\left(x_{1}, x_{2}, x_{3}\right), x_{4}\right)$;

(б) если $f((x, y, z), z)=0$, mo $f((x, y, z), t)=0$;

(в) если $f(x, y)$ - дифференцирование по $x, y$ и $f((x, y, z), z)=0$, то на алгебре $S$ выполнено тождество $f(x, y)=0$.

ДокАЗАТЕЛЬСтво. (а) Используя свойства функции $f(x, y)$, перечисленные в замечании, получаем

$$
\begin{aligned}
f((x, y, z), t)=f((x y) z, t)-f(x(y z), t)=f(x y, z t)+f(z,(x y) t)-f(y z, x t) \\
\quad-f(x,(y z) t)=\{f(x, y(z t))+f(y, x(z t))\}+f(z,(x y) t) \\
\quad-\{f(y, z(x t))+f(z, y(x t))\}-f(x,(y z) t)=\{f(x, y(z t))-f(x,(y z) t)\} \\
\quad+\{f(y, x(z t))-f(y, z(x t))\}+\{f(z,(x y) t)-f(z, y(x t))\} \\
=-f(x,(y, z, t))+f(y,(z, t, x))+f(z,(y, x, t)) \\
=f((t, y, z), x)+f((x, t, z), y)+f((x, y, t), z) .
\end{aligned}
$$

(б) Допустим теперь, что $f((x, y, z), z)=0$. Тогда функция $f((x, y, z), t)$ кососимметрична относительно указанных переменных и из п. (а) получаем

$$
f((x, y, z), t)=f((t, y, z), x)+f((x, t, z), y)+f((x, y, t), z)=3 f((x, y, z), t)=0 .
$$

(в) Наконец, если $f(x, y)$ - дифференцирование и $f((x, y, z), z)=0$, то в силу п. (б) $f((x, y, z), t)=0$, т.е. $f(V, S)=(0)$, где $V$ - подпространство, порожденное всеми ассоциаторами алгебры $S$. Тогда $f\left(V^{2}, S\right)=(0)$ и, значит, $f(W, S)=(0)$, где $W$ - идеал алгебры $S$, порожденный множеством $(V, S, S)$ (см. [6]). Учитывая, что $f$ - дифференцирование, получаем: $f(S, S)$ содержится в аннуляторе ненулевого идеала $W$, откуда в силу первичности $S$ получаем требуемое утверждение. 


\section{2. О некоторых центрах первичных алгебр.}

Лемма 11. Ассоииативный чентр $N_{\mathrm{Ass}}[S]$ алгебры $S$ нулевой.

ДоказАТЕЛЬство. Если $p \in N_{\mathrm{Ass}}[S]$, то идеал алгебры $S$, порожденный элементом $p$, имеет вид $(p)=\Phi p+p S$, значит, $(p)^{2}=\left(p^{2}\right)$ и $(p)^{3}=(0)$. Так как первичная алгебра не имеет нильпотентных идеалов, то $p=0$.

ОПРЕДЕЛЕНИЕ. Пусть $P_{x}(y, z, t):=((y, x, z), x, t)$. Центром $N_{P}[S]$ функuии $P$ в алгебре $S$ назьвается множество

$$
N_{P}[S]:=\left\{p \in S: P_{x}(p, y, z)=0 \quad \forall x, y, z \in S\right\} .
$$

СлеДСТвИЕ. Центр $N_{P}[S]$ алгебры $S$ нулевой.

ДокаЗАТЕЛЬСтво. Заметим, что функция $f(x, y):=(x, y, p)$, где $p \in N_{P}[S]$, удовлетворяет всем условиям леммы 10 (в), следовательно, $(x, y, p)=0$ для любых элементов $x, y \in S$, т.е. $p \in N_{\mathrm{Ass}}[S]$, и ввиду леммы 11 имеем $p=0$.

ОПреДЕлЕНИЕ. Центром $N_{G}[S]$ функции $G$ в алгебре $S$ называется множество:

$$
N_{G}[S]:=\left\{p \in S: G_{x}(p, y, z, t, v)=0 \quad \forall x, y, z, t, v \in S\right\} .
$$

Лемма 12. Центр $N_{G}[S]$ алгебры $S$ нулевой.

ДокаЗАТЕЛЬСТво. Пусть $p \in N_{G}[S]$, т.е. на основании леммы 3 для любых $x, y, z, t, v \in S$ справедливы равенства:

$$
\begin{aligned}
p R(y, x) R(z, x) R(t, v) & =p R(y, z) R(x, t) R(x, v), \\
(((p, y, x), z, x), t, v) & =(((p, y, z), x, t), x, v) .
\end{aligned}
$$

После линеаризации тождества (4) подстановкой $x \rightarrow x^{2}$ на основании тождеств $\left(a, b^{2}, c\right)=(a, b, b \circ c)=(a, b, c) \circ b$ получаем

$$
(((p, y, x), z, x) \circ x, t, v)=(((p, y, z), x, t), x, v) \circ x,
$$

следовательно,

$$
((p, y, x), z, x)(x, t, v)=0 .
$$

Далее, оператор $R(a, b)$ является дифференцированием алгебры $S$, значит, центр функции $G$ замкнут относительно оператора $R(a, b)$, т.е.

$$
N_{G}[S] R(a, b) \subseteq N_{G}[S] .
$$

Учитывая соотношения (4) и (7), имеем

$$
\begin{gathered}
N_{G}[S] R(y, x) R(z, x) R(t, v)=N_{G}[S] R(y, z) R(x, t) R(x, v) \subseteq N_{G}[S] R(t, x) R(v, x), \\
\text { следовательно, полагая } M(x):=\left(\left(N_{G}, S, x\right), S, x\right), \text { имеем } \\
M(x) R(S, S) \subseteq M(x) .
\end{gathered}
$$


Далее, тождество (6) в этих обозначениях принимает вид

$$
M(x) \cdot(x, S, S)=(0)
$$

Поскольку $R(a, x) R(b, x) R(c, x)=0$ (см. [4; лемма 2]), то

$$
(M(x), S, x)=(0)
$$

и для любого $m \in M(x)$ имеем:

$$
(m, a,(x, b, c))=((m, a, x), b, c)-((m, b, c), a, x)-(m,(a, b, c), x)=0
$$

откуда

$$
(M(x), S,(x, S, S))=(0) .
$$

Заметим теперь, что в силу $(8) M(x)+M(x) S$ является идеалом алгебры $S$, причем на основании (9) и (10) это идеал с нулевым умножением, значит, он равен нулю, т.е. $\left(\left(N_{G}, S, x\right), S, x\right)=(0)$. Отсюда $N_{G}[S] \subseteq N_{P}[S]$. Поскольку $N_{P}[S]=(0)$ ввиду следствия из леммы 11 , то центр $N_{G}[S]$ также нулевой.

\section{3. Редукция от функции $G$ к ассоциатору.}

ЛЕмма 13. Допустим, что $a, b$ - такие әлементы алгебры S, что для любых әлементов $x, y, z, t$ алгебры $S$ верно равенство $G_{x}(a, b, y, z, t)=0$. Тогда $(a, b, S)=(0)$.

ДокАЗАТЕЛЬСтво. Рассмотрим функцию

$$
f_{1}\left(\xi_{1}, \xi_{2}, \ldots, \xi_{5}\right):=G_{x}\left(\left(a, b, \xi_{1}\right), \xi_{2}, \ldots, \xi_{5}\right)
$$

Докажем, что эта функция кососимметрична по переменным типа $\xi$. Для этого достаточно понять, что она обрашается в нуль при $\xi_{1}=\xi_{2}=\xi$.

Вначале заметим, что

$$
G_{x}((r, s, y), r, s, z, t)=0 ;
$$

действительно, в силу муфанговости функции $G_{x}$ имеем

$$
\begin{aligned}
G_{x}((r, s, y), r, s, z, t) & =-G_{x}((r, y, s), r, s, z, t) \\
& =-G_{x}((r y) s, r, s, z, t)+G_{x}(r(y s), r, s, z, t) \\
& =-G_{x}(r y, r, s, z, s t)+G_{x}(y s, r, s, z r, t) \\
& =-G_{x}(y, r, s, z r, s t)+G_{x}(y, r, s, z r, s t)=0 .
\end{aligned}
$$

Из (11) вытекает кососимметричность функции $G_{x}((p, q, \xi), \xi, r, z, t)$ по переменньмм $p, q, r, z, t$, следовательно,

$$
\begin{aligned}
G_{x}((a, b, \xi), \xi, y, z, t) & =-G_{x}((y, b, \xi), \xi, a, z, t) \\
& =G_{x}((y, z, \xi), \xi, a, b, t) \in G_{x}(a, b, S, S, S)=(0) .
\end{aligned}
$$

Тем самым доказана кососимметричность функции $f_{1}\left(\xi_{1}, \xi_{2}, \ldots, \xi_{5}\right)$ по переменным типа $\xi$. 
Далее, учитывая, что оператор $R(r, s)$ является дифференцированием алгебры $S$, получаем также (ввиду условия леммы) симметричность функции $G_{x}\left((a, r, s), b, \xi_{3}, \xi_{4}, \xi_{5}\right)$ по переменным $a, b$. Поскольку функция $f_{2}(p, q):=$ $G_{x}\left(p, q, \xi_{3}, \xi_{4}, \xi_{5}\right)$ является слабо муфанговой, то в силу леммы 10 (а) верно равенство:

$$
f_{2}((a, r, s), b)=f_{2}((b, r, s), a)+f_{2}((a, b, s), r)+f_{2}((a, r, b), s)
$$

из которого в силу симметричности $f_{2}((a, r, s), b)$ по переменным $a, b$ имеем

$$
f_{2}((a, b, s), r)+f_{2}((a, r, b), s)=0
$$

т.е. функция $f_{2}((a, b, s), r)$ симметрична по $r, s$. Итак, функция

$$
G_{x}\left(\left(a, b, \xi_{1}\right), \xi_{2}, \xi_{3}, \xi_{4}, \xi_{5}\right)
$$

одновременно симметрична и кососимметрична по переменным $\xi_{1}$ и $\xi_{2}$, значит, она является нулевой. Таким образом, $(a, b, S)$ содержится в центре $N_{G}[S]$ и, значит, в силу леммы 12 получаем требуемое утверждение.

\section{4. Опровержение тождеств типа $G, F, H$.}

ТЕОРема 1. В первичной исключительной коммутативной альтернативной алгебре $S$ все функиии типа $G, F, H$ являются ненулевыми.

ДоказАтЕЛьство. Достаточно, очевидно, доказать утверждение только для функций типа $H$. Итак, допустим, что $H(3 n+8)=0$ - тождество алгебры $S$.

Тогда $F\left(B_{n}, a, c \mid P_{2 n}, x, y, z, t, d\right) R(d, v)=0$ и, вводя дифференцирование $r D:=F\left(B_{n}, a, c \mid P_{2 n}, x, y, z, t, r\right)$, получаем в силу тождества $(r D, r, s)=0$ :

$$
\begin{aligned}
(q, r, s) D & =(q D, r, s)+(q, r D, s)+(q, r, s D) \\
& =(q D, r, s)-(r, q D, s)-(s, r, q D)=3(q D, r, s)=0 .
\end{aligned}
$$

Следовательно, рассуждая аналогично доказательству леммы 10 (в), имеем

$$
F\left(B_{n}, a, c \mid P_{2 n}, x, y, z, t, W(S)\right)=(0), \quad \text { т.е. } F\left(B_{n}, a, c \mid P_{2 n}, x, y, z, t, d\right)=0 .
$$

Последнее равенство принимает вид $G_{a}\left(G\left(B_{n} \mid P_{2 n}, x, y, c\right), c, z, t, v\right)=0$. Тогда в силу леммы 13 получаем $G\left(B_{n} \mid P_{2 n}, x, y, c\right) R(c, z)=0$. Применяя теперь для дифференцирования $r D:=G\left(B_{n} \mid P_{2 n}, x, y, r\right)$ аналогичные рассуждения, приходим к равенству $G\left(B_{n} \mid P_{2 n}, x, y, W(S)\right)=(0)$, т.е. $G\left(B_{n} \mid P_{2 n}, x, y, z\right)=0$.

Используя лемму 2 , по индукции легко проверить, что фуннкция $G(3 n+3)$ допускает представление:

$$
G\left(B_{n} \mid P_{2 n+3}\right)=p_{1} \nabla_{p_{2}}\left(p_{3}, p_{4}, b_{1}\right) \cdot\left(\prod_{i=2}^{n} \nabla_{b_{i-1}}\left(p_{2 i+1}, p_{2 i+2}, b_{i}\right)\right) R\left(b_{n}, p_{2 n+3}\right) .
$$

Тогда на основании той же леммы 2 имеем

$$
G_{b_{n}}\left(Q_{n-1}, b_{n-1}, p_{2 n+1}, p_{2 n+2}, p_{2 n+3}\right)=0
$$


где

$$
Q_{n-1}=p_{1} \nabla_{p_{2}}\left(p_{3}, p_{4}, b_{1}\right) \cdot\left(\prod_{i=2}^{n-1} \nabla_{b_{i-1}}\left(p_{2 i+1}, p_{2 i+2}, b_{i}\right)\right) .
$$

Применяя теперь лемму 13 , получаем равенство $\left(Q_{n-1}, b_{n-1}, p_{2 n+1}\right)=0$, которое в принятых обозначениях имеет вид $G\left(B_{n-1} \mid P_{2 n+1}\right)=0$. Тем самьм, по индукции получаем, что в алгебре $S$ выполнено тождество $G(6)=0$, но тогда алгебра $S$ в силу леммы 13 должна быть ассоциативна. Полученное противоречие завершает доказательство теоремы.

\section{§4. Цепочки многообразий}

4.1. Формулировка основных результатов. Пусть, как и прежде, $\Phi$ - поле характеристики $3 ; \operatorname{Alt}_{n} \Phi$ - многообразие $\Phi$-алгебр, порожденное свободной альтернативной $\Phi$-алгеброй ранга $n$.

ТЕОРЕМА 2. Пусть $V$ - произвольное многообразие альтернативных алгебр над полем Ф характеристики 3 , содержащее хотя бъ одну исключительную первичную коммутативную алгебру. Тогда в чепочке многообразий

$$
V_{8} \subset V_{9} \subset \cdots \subset V_{n} \subset \cdots
$$

все включения строгие.

Tеорема 3. В иепочке многообразий $\operatorname{Alt}_{4} \Phi \subset \cdots \subset \operatorname{Alt}_{n} \Phi \subset \cdots$ альтернативных алгебр над полем $\Phi$ характеристики 3 все включения строгие.

ОСНовная теоремА. $B$ иепочке многообразий $\operatorname{Alt}_{1} \Phi \subset \cdots \subset \operatorname{Alt}_{n} \Phi \subset \ldots$ альтернативных алгебр над полем $\Phi$ характеристики 3 все включения строzue.

4.2. Доказательство теорем. Проше всего доказать теорему 2. Из леммы 9 и теоремы 1 вытекает, что функции $G(3 n+3), F(3 n+4), H(3 n+5) \quad(n \geqslant 2)$ являются нетривиальными тождествами $V$-свободных алгебр ранга $3 n+2,3 n+3$, $3 n+4$ соответственно.

ДоКАЗАТЕЛЬСтво тЕОРемы 3. Заметим, что в силу теоремы 2 и сушествования исключительной первичной коммутативной альтернативной $\Phi$-алгебры в цепочке многообразий $\mathrm{Alt}_{4} \subset \mathrm{Alt}_{5} \subset \cdots \subset \mathrm{Alt}_{n} \subset \cdots$ все включения строгие, за исключением, быть может, начального отрезка $\mathrm{Alt}_{4} \subset \cdots \subset \mathrm{Alt}_{8}$. Для доказательства строгости включений на этом отрезке достаточно проверить утверждения:

а) функция $g(5)$ различает многообразия $\mathrm{Alt}_{4}$ и $\mathrm{Alt}_{5}$;

б) функция $G(6)$ различает многообразия $\mathrm{Alt}_{5}$ и $\mathrm{Alt}_{6}$;

в) функция $G_{a}(x, y, z, t, p) R(p, v)$ различает многообразия $\mathrm{Alt}_{6}$ и $\mathrm{Alt}_{7}$;

г) функция $g(8)$ различает многообразия $\mathrm{Alt}_{7}$ и $\mathrm{Alt}_{8}$.

Указанные функции, как следует из результатов $\S 2$, на самом деле являются тождествами свободных альтернативных алгебр соответствующего ранга, например $g(8)=0$ - тождество в алгебре ранга 7. Осталось убедиться, что эти четыре функции не являются тождествами свободной альтернативной алгебры. 
Для функций из пш. б) и в) это утверждение было доказано в 3 . Покажем, что функция $g(8)$ принимает ненулевые значения на свободной центрально метабелевой альтернативной алгебре $F$. Итак, допустим от противного, что $g(8)=0$ на алгебре $F$ (напомним, что алгебра $F$ называется иентрально метабелевой, если $\left.\left(F^{2}\right)^{2} \subseteq \operatorname{Ann} F\right)$. Тогда

$$
[x, a]\{y, z, a\}_{b}\{p, q, b\}_{s}=0
$$

Пусть $v, w \in F^{2}$. Поскольку по модулю Ann $F$ имеем $p(a b) p=(p a)(b p)=0$ (ввиду центрального тождества Муфанг), то

$$
\begin{aligned}
v D(p, q) & =(v \circ p) \circ q-(v \circ q) \circ p=2(v \circ p) \circ q=2(v \circ p) q+2 q(v \circ p) \\
& =2 p(v q)+2(q v) p=2(q v) p-2 q(v p)=2(q, v, p)=2(v p) q .
\end{aligned}
$$

Вьполнив линеаризацию $a \rightarrow v$ тождества (12), получим

$$
\begin{aligned}
0 & =[x, v]\{y, z, a\}_{b}\{p, q, b\}_{s} \\
& =2[x, v]\{R(y, z) R(a, b)+R(a, z) R(y, b)+R(y, a) R(z, b)\}\{p, q, b\}_{s} \\
& =2[x, v] R(y, a) R(z, b)\{p, q, b\}_{s}
\end{aligned}
$$

линеаризация по $b \rightarrow w$ последнего тождества дает:

$$
\begin{aligned}
0 & =2[x, v] R(y, a) R(z, b)\{p, q, w\}_{s} \\
& =[x, v] R(y, a) R(z, b)\{R(p, q) R(w, s)+R(w, q) R(p, s)+R(p, w) R(q, s)\} \\
& =[x, v] R(y, a) R(z, b) R(p, q) R(w, s) .
\end{aligned}
$$

Следовательно,

$$
\left[F^{2}, F\right] R^{6}(F) \cdot F^{3}=(0)
$$

Однако, как следует из [7], последнее невозможно. Полученное противоречие завершает доказательство теоремы 3.

Основная теорема вытекает из теоремы 3 и классических результатов Артина, Дорофеева, Хумм и Клейнфельда (см. [3]).

\section{§5. Нильпотентность разрешимых алгебр из многообразий $\mathrm{Alt}_{n}$}

ЛЕмма 14. Пусть $A-$ свободная конечно порожсденная альтернативная алгебра над произвольным полем ранга $n ; f(x, y, z, t)$ - муфангова функиия на алгебре $A$, записанная в виде линейной комбинации ассоциаторов; $R^{*}(A, A)$ - подалгебра алгебры умножсений, порожсенная операторами $R(a, b)$. Тогда алгебра $R^{*}(A, A)$ действует нильпотентно на идеале $(f)$, порожсенном функцией $f$. 
ДокАЗАТЕЛЬСТво. Докажем сначала тождество

$$
(f(a, b, x, y), a, b)=0 .
$$

Используя кососимметричность ассоциатора, преобразуем выражение в левой части

$$
\begin{aligned}
(f(a, b, x, y), a, b) & =-(a, f(a, b, x, y), b) \\
& =-f(a, b, x, y) L(a) R(b)+f(a, b, x, y) R(b) L(a) .
\end{aligned}
$$

Преобразуем теперь каждое из полученных слагаемых, применяя муфанговость функции $f$ :

$$
\begin{aligned}
& f(a, b, x, y) L(a) R(b)=f(a, b, x a, y) R(b)=f(a, b, x a, b y), \\
& f(a, b, x, y) R(b) L(a)=f(a, b, x, b y) L(a)=f(a, b, x a, b y) .
\end{aligned}
$$

Тем самым тождество (13) доказано.

Аналогично тождеству (13) проверяется, что $f(a, b, x, y)[R(a), R(b)]=0$ :

$$
f(a, b, x, y) R(a) R(b)=f(a, b, a x, b y)=f(a, b, a x, y) R(b) R(a) .
$$

Отсюда ввиду тождества (13) имеем

$$
f(a, b, x, y)[a, b]=0,
$$

поскольку $f(a, b, x, y)[a, b]=f(a, b, x, y)[R(a), R(b)]=0$.

Далее, из тождества (14) вытекает

$$
f(a, b, x, y) \cdot[a, b]^{4}=0 .
$$

Последовательная линеаризация тождества (15) по переменной $a$, а затем по переменной $b$ приводит к тождествам вида:

$$
\begin{gathered}
f(r, b, x, y) \cdot[a, b]^{4}+f(a, b, x, y) \cdot w_{r}(a, b)=0, \\
f(r, s, x, y) \cdot[a, b]^{4}+f(r, b, x, y) \cdot w_{s}(b, a) \\
+f(a, s, x, y) \cdot w_{r}(a, b)+f(a, b, x, y) \cdot w_{r, s}(a, b)=0
\end{gathered}
$$

где $w_{r}(a, b)=\Delta_{a}^{1}(r)[a, b]^{4}$ и $w_{r, s}(a, b)=\Delta_{b}^{1}(s) w_{r}(a, b)$.

Поскольку функции $[a, b]^{4}, w_{r}(a, b), w_{r, s}(a, b)$ содержатся в ассоциативном центpe $N_{\text {Ass }}[A]$ и перестановочны с произвольным ассоциатором, то справедливы равенства:

$$
\begin{aligned}
& f(r, b, x, y) \cdot[a, b]^{4}=w \cdot f(a, b, x, y), \\
& f(r, s, x, y) \cdot[a, b]^{4}=\sum_{i} w_{i} \cdot f(u, v, x, y),
\end{aligned}
$$


где $u \in\{a, b\}, w, w_{i} \in N_{\mathrm{Ass}}[A]$. Используя тождества $(16)$ и $(17)$, получаем для некоторого $w \in N_{\mathrm{Ass}}[A]$

$$
f(r, s, x, y) \cdot[a, b]^{8}=w \cdot f(a, b, x, y)
$$

Откуда в силу (15) имеем

$$
f(r, s, x, y) \cdot[a, b]^{12}=0
$$

Напомним, что во всякой альтернативной алгебре $A$ для элемента $w \in N_{\mathrm{Ass}}[A]$ справедливы утверждения (см. [3; гл. $7, \S \S 1,2])$ :

а) если $w \in N_{\mathrm{Ass}}[A]$, то $\operatorname{Ideal}(w)=A^{\#} w A^{\#}$;

б) $[w, A] \in N_{\mathrm{Ass}}[A]$;

в) если $w \in N_{\mathrm{Ass}}[A]$, то $[w,(A, A, A)]=(0)$.

Положим для сокращения записи

$$
f:=f(r, s, x, y), \quad w:=[a, b]^{12}
$$

и проверим справедливость тождества:

$$
f\left(p w^{2} q\right)=0
$$

Учитывая (18) и приведенные утверждения об ассоциативном центре, последовательно получаем:

$$
\begin{aligned}
f\left(w^{2} p\right) & =\left(f w^{2}\right) p=0, \\
f\left[p, w^{2}\right] & =f(w \circ[p, w])=(f w)[p, w]+(f[p, w]) w=([p, w] f) w=[p, w](f w)=0, \\
f\left(p w^{2}\right) & =f\left(w^{2} p\right)+f\left[p, w^{2}\right]=0, \\
\left(f, p w^{2}, q\right) & =\left(f, w^{2} p, q\right)=\left(f\left(w^{2} p\right)\right) q-f\left(\left(w^{2} p\right) q\right)=-f\left(\left(w^{2} p\right) q\right) \\
& =-f\left(w^{2}(p q)\right)=0 \\
f\left(p w^{2} q\right) & =\left(f\left(p w^{2}\right)\right) q=0 .
\end{aligned}
$$

Отсюда вытекает равенство $f \cdot\left(w^{2}\right)=(0)$ и, аналогично, $\left(w^{2}\right) \cdot f=(0)$, следовательно, идеал $\left(w^{2}\right)$ содержится в аннуляторе идеала $(f)$. Применив теперь теорему Шестакова [8] об ассоциаторной нильпотентности конечно порожденных альтернативных алгебр с тождеством $[x, y]^{m}=0$, получим требуемое в лемме утверждение.

TеоРемА 4. В многообразии $\mathrm{Alt}_{n}$ альтернативных алгебр конечного базисного ранга над полем характеристики 3 всякая разрешимая алгебра нильпотентна.

ДокАЗАТЕЛьство. В силу редукции Дорофеева [9] теорему достаточно доказать для произвольной метабелевой (разрешимой индекса 2) алгебры из многообразия $\mathrm{Alt}_{n}$. Пусть $B$ - метабелева алгебра из многообразия $\mathrm{Alt}_{n}$. По лемме 14 существует число $N$, зависящее только от ранга $n$, такое, что в многообразии Alt $n$ справедливо тождество

$$
G_{x}(y, z, t, u, v) R\left(a_{1}, a_{2}\right) \cdots R\left(a_{2 N-1}, a_{2 N}\right)=0
$$


Тогда после линеаризации по $x$ имеем в алгебре $B$ :

$$
\begin{aligned}
0 & =G_{r s, x}(y, z, t, u, v) R\left(a_{1}, a_{2}\right) \cdots R\left(a_{2 N-1}, a_{2 N}\right) \\
& =(y, z, r s) D(x, t) R(u, v) R\left(a_{1}, a_{2}\right) \cdots R\left(a_{2 N-1}, a_{2 N}\right) \\
& =2((r s, y), x, t) R(u, v) R\left(a_{1}, a_{2}\right) \cdots R\left(a_{2 N-1}, a_{2 N}\right) \\
& =2(r s) R(y) R(z) R(x) R(t) R(u) R(v) R\left(a_{1}\right) R\left(a_{2}\right) \cdots R\left(a_{2 N-1}\right) R\left(a_{2 N}\right),
\end{aligned}
$$

следовательно, алгебра $B$ нильпотентна.

Теорема 4 для альтернативных алгебр над полем характеристики 0 доказана И.П. Шестаковым [1], ее аналог для алгебр характеристики, отличной от 2 и 3 , был получен В. Т. Филипповым [2].

\section{Список литературы}

1. Шестаков И. П. Об одной проблеме Ширшова // Алгебра и логика. 1977. Т. 16. № 2. C. $227-246$.

2. Филиппов В. T. О цепочках многообразий, порожденныт свободными мальцевскими и альтернативньми алгебрами // Докл. АН СССР. 1981. Т. 260. № 5. С. 1082-1085.

3. Жевлаков К.А., Слинько А.М., Шестаков И.П., Шириов А. И. Кольца, близкие к ассоциативньм. М.: Наука, 1978.

4. Пчелинцев C. В. О кручении свободного альтернативного кольца // Сиб. матем. журн. 1991. T. 32. №6. C. $142-149$.

5. Пчелинцев C. В. О нильпотентных элементах и ниль-радикалах альтернативных алгебр // Алгебра и логика. 1985. Т. 24. №6. С. 674-695.

6. Пчелинцев С. В. Теорема о высоте для альтернативных алгебр // Матем. сб. 1984. T. 124. № 4. C. 557-567.

7. Пчелинцев C. B. Разрешимость и нильпотентность альтернативных алгебр и алгебр типа $(-1,1)$ // Групшы и другие алгебраические системы с условиями конечности. Новосибирск: Наука, 1984. С. 81-101.

8. Шестаков И. П. Альтернативные алгебры с тождеством $[x, y]^{m}=0 / /$ Алгебра и логика. 1981. Т. 20. № 5. С. 575-596.

9. Дорофеев Г. В. О локально нильпотентном радикале неассоциативных колец // Алгебра и логика. 1971. Т. 10. № 4. С. 355-364.

Московский городской педагогический университет

Поступила в редакцию

17.10 .2000 